\title{
Spatial frequency-selective losses with pattern electroretinogram in Type 1 (insulin-dependent) diabetic patients without retinopathy
}

\author{
M.A.S. Di Leo ${ }^{1}$, B. Falsini ${ }^{2}$, S. Caputo ${ }^{1}$, G. Ghirlanda ${ }^{1}$, V.Porciatti ${ }^{3}$ and A. V. Greco ${ }^{1}$ \\ Departments of ${ }^{1}$ Internal Medicine and ${ }^{2}$ Ophthalmology, Catholic University, Rome \\ ${ }^{3}$ Institute of Neurophysiology, National Council of Research, Pisa, Italy
}

\begin{abstract}
Summary. Neurosensory abnormalities have been implicated in the first stages of diabetic retinopathy. The activity of retinal ganglion cells in 24 Type 1 (insulin-dependent) diabetic patients with short disease duration without retinopathy on fluorescein angiography was investigated by using a pattern electroretinogram in response to sinusoidal gratings of different spatial frequencies $(0.6,1.0,1.4,2.2$, $4.8 \mathrm{cycles} / \mathrm{deg}$ ), counterphase modulated at $8 \mathrm{~Hz}$. The pattern electroretinogram reflects, at least in part, the activity of subsets of generators (i.e., ganglion cells) which show spatial selectivity. Mean pattern electroretinogram amplitude was significantly reduced in patients at lower and intermediate,
\end{abstract}

but not at higher spatial frequencies compared with 40 agematched control subjects. At 1.4 cycles/deg the pattern electroretinogram amplitude was significantly correlated $(r=0.59)$ with age at onset $(p=0.002)$ and duration of disease $(p=0.002)$. Our results suggest that in Type 1 diabetic patients without retinopathy, there is an early sensory deficit of specific inner retina neurons which respond preferentially to gratings of medium and large size.

Key words: Retinal function, ganglion cells, pattern electroretinogram, spatial frequency, diabetic retinopathy.
Type 1 (insulin-dependent) diabetic patients may exhibit visual dysfunctions which can be detected by means of psychophysical (contrast sensitivity) [1-3] and electrophysiological (visual evoked potentials (VEPs) and electroretinogram (ERG) [4-9] techniques. Stimuli generally employed in clinical tests are periodical patterns (gratings or checkerboards) of variable spatial frequency (i.e., the number of light-dark cycles subtended in one degree of visual angle) which are presented either statically or dynamically (i.e., alternating at constant mean luminance) at threshold (for contrast sensitivity testing) or suprathreshold (for VEPs and ERG recording) contrast. Neurosensorial deficits have been reported as affecting responses to a restricted range of spatial frequencies, indicating a selective vulnerability of the visual system to diabetes. The primary site of metabolic and/or vascular damage in diabetes is probably located in the postreceptoral retina [5].

The ERG evoked by alternating gratings reversing in contrast at a constant mean luminance (pattern ERG, PERG) had been clinically employed to evaluate macular function $[10,11]$. P-ERG is a focal response that is thought to be correlated, unlike the more conventional Iuminance-evoked (flash) ERG, with ganglion cell activity [10, 12]. Experimental [13] and clinical $[11,14,15]$ studies re- cently demonstrated that P-ERG was altered in cases of ganglion cell dysfunctions, whereas the flash-ERG was normal. The P-ERG technique may therefore provide a direct index of inner retina function of directly stimulated retinal area. P-ERG properties depend on the spatial characteristic of the stimulus [11]. In particular, the response displays spatial selectivity with a maximum amplitude at intermediate spatial frequencies and attenuation at higher and lower ones $[10-12,16]$. This response pattern distinguishes, at least in part, different subpopulations of retinal ganglion cells which preferentially respond to specific spatial stimuli.

To evaluate the integrity of the ganglion cell subsystems in Type 1 diabetic patients without retinopathy, $\mathrm{P}$ ERG as a function of spatial frequency was used.

\section{Subjects and methods}

\section{Subjects}

The study included 24 Type 1 diabetic patients. The patients (11 men, 13 women) aged from 11 to 29 years (mean \pm SEM, $19.5 \pm 0.6$ years) and showed no retinopathy. The average disease duration was $3.4 \pm 0.3$ years. The mean $\mathrm{HbA}_{1 \mathrm{c}}$ value was $6.5 \pm 0.2 \%$ 


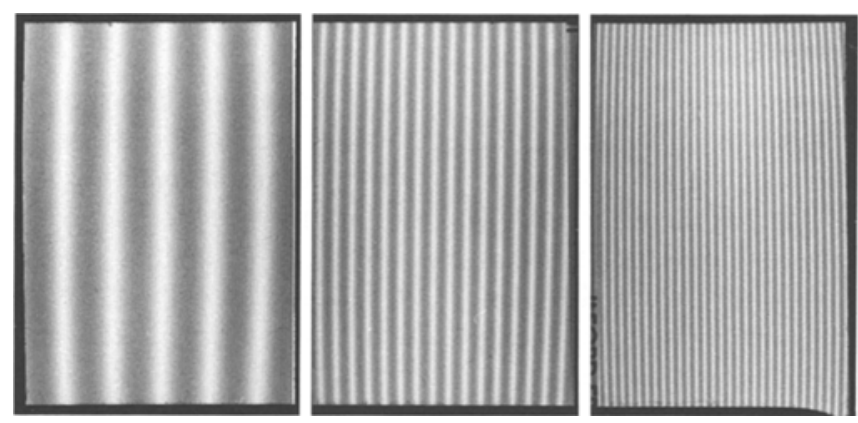

Fig. 1. Grating detection tests of variable spatial frequencies are demonstrated. Left to right: patterned stimuli shown on a television monitor consist of sinusoidal gratings of low, intermediate and high spatial frequencies. Note that the number of light and dark bars is less at lower spatial frequencies than at higher ones

(range 4-8.4\%). Forty healthy subjects ( 16 men, 24 women) with an age (21.8 \pm 0.8 years) and sex distribution comparable to that of the diabetic patients served as the control group.

Before inclusion in the study, all patients received a general ocular examination including direct and indirect ophthalmoscopy and slit-lamp biomicroscopy. Colour fundus photographs and fluorescein angiography of both eyes were also performed. None had other ocular or systemic diseases or were receiving medication. No retinopathy and/or macular oedema were found in any case. The best-corrected visual acuity was $20 / 20$ or better. Refractive errors, when present, were below \pm 1.00 diopters, and were fully corrected during the P-ERG recording. Diabetic patients were tested after eating to avoid hypoglycaemia. Informed consent was obtained from each subject after the nature of the test and the study were fully explained.

\section{Methods}

Fluorescein angiography was performed with a Kowa (Pro $150^{\circ}$ ) fundus camera after rapid injection of $5 \mathrm{ml}$ of $10 \%$ fluorescein sodium into the antecubital vein. Angiograms were taken with ASA 400 black-and-white film.

Visual stimulus consisted of light and dark vertical bars with a sinusoidal profile at different spatial frequencies $(0.6,1.0,1.4,2.2$, and 4.8 cycles $/ \mathrm{deg}$; fixed contrast, $56 \%$ ). Gratings were generated electronically on a high-resolution television monitor (stimulus field, $24 \times 14 \mathrm{~cm}$; mean luminance, $100 \mathrm{~cd} / \mathrm{m}^{2}$ ) and modulated (sinusoidally) in counterphase at a temporal frequency of $8 \mathrm{~Hz}$ (Fig.1). The monitor was peripherally covered by a large equiluminant white cardboard $(70 \times 70 \mathrm{~cm})$ in a way that left a central rectangular portion that represented the stimulating field. Subjects with natural pupils, monocularly maintained fixation on the centre of the stimulating field from a $57 \mathrm{~cm}$ viewing distance. Pupil sizes were measured and no differences were observed between diabetic patients and control subjects. P-ERGs were recorded by a small silver disc taped over the skin of the lower eyelid. An identical electrode placed over the contralateral eyelid of the unstimulated eye was used as a reference (interocular P-ERG [12]). Retinal signals were bandpass filtered between 1 and $30 \mathrm{~Hz}$ ( $-6 \mathrm{~dB} / \mathrm{oct}$ ), amplified 100,000-fold and averaged (more than 300 events) by an IBM PC/XT computer (12 bit resolution; $0.5 \mathrm{~ms}$ sampling rate) with rejection of single sweeps disturbed by artifacts (blinks, eye movements, or head movements). Fourier analysis (Discrete Fourier Series [17]) of the averaged response was performed off-line by the same processor to mathematically isolate the main harmonic component (second harmonic: twice the stimulation frequency). The peak-to-peak amplitude of this component was measured in microvolts and plotted as a function of the stimulus spatial frequency. Each test was repeated twice; the average amplitude variation between the two records was $5 \%$ for both control subjects and diabetic patients. The average bio- logical noise at the reversal frequency, obtained with the monitor covered by a piece of white cardboard, was $0.09 \pm 0.05 \mu \mathrm{V}$.

A fasting blood sample to measure $\mathrm{HbA}_{\mathrm{c}}$ values was drawn from each patient in the morning. $\mathrm{HbA}_{1 c}$ was assayed by $\mathrm{HPLC}$ with $5.8 \%$ as the upper limit of the normal range.

\section{Statistical analysis}

Results are presented as means \pm SEM. Before statistical analysis of the results, we considered only the right eye P-ERG amplitudes in control subjects, because the intereye correlation of the latter subjects was significant. On the contrary, the intereye correlation in our diabetic population was not significant for each spatial frequency. We therefore used a two-eye statistical analysis according to Ray and O'Day [18]. In the normal group, we confirmed by normality test the normal distribution of P-ERG amplitude data. Statistical evaluation of the data was performed with the correlation between the two eyes of a subject, unpaired Student's $t$-test for comparing the age and sex of the groups, one-way analysis of variance (ANOVA), and multiple regression analysis.

\section{Results}

There was no significant difference between the diabetic and control groups for sex and age at the time of the study.

Figure 2 (left column) shows representative examples of steady state $\mathbf{P}$-ERG recorded in a normal subject at different spatial frequencies. The P-ERG waveform is approximately sinusoidal with a temporal period of about $62 \mathrm{~ms}$. Figure 2 (right column) shows the Discrete Fourier Series of each response. It can be noted that the height of each vertical bar $(n=20)$ of the Fourier analysis display indicates the relative contribution (in \%) of the corresponding harmonic component for the peak-to-peak signal. The response component at twice the stimulation frequency (2nd harmonic: 2 nd vertical bar from left) yields the major contribution to P-ERG at each measured frequency.

Figure 3 shows the individual P-ERG responses for each spatial frequency in diabetic patients, the normal range area, and means of control and diabetic subjects. Mean P-ERG amplitudes of control subjects are spatially tuned with a clear maximum at $1.4 \mathrm{cycles} / \mathrm{deg}$. In diabetic patients P-ERG responses were significantly reduced at $0.6 \mathrm{cycles} / \mathrm{deg}$ (one-way ANOVA: $\mathrm{F}$ test $=9.7 ; 0.59 \pm 0.03$ vs $0.72 \pm 0.03 \mu \mathrm{V} ; \mathrm{df}=1,46 ; p=0.002), 1.0$ cycles $/ \mathrm{deg}(\mathrm{F}$ test $=17.3 ; 0.61 \pm 0.04$ vs $0.81 \pm 0.03 \mu \mathrm{V} ; p=0.0001$ ), 1.4 cycles $/$ deg $(\mathrm{F}$ test $=27.8 ; 0.61 \pm 0.03$ vs $0.85 \pm 0.03 \mu \mathrm{V}$; $p=0.0001)$ and 2.2 cycles $/$ deg $(\mathrm{F}$ test $=6 ; 0.65 \pm 0.04 \mathrm{vs}$ $0.76 \pm 0.02 \mu \mathrm{V} ; p=0.02$ ) spatial frequencies as compared with those of control subjects. At 4.8 cycles/deg there was no significant difference $(0.52 \pm 0.03$ vs $0.58 \pm 0.03 \mu \mathrm{V})$ between diabetic and control subjects.

Multiple regression analysis was performed with $\mathrm{P}$ ERG amplitude for each spatial frequency as the dependent variable and age at onset, duration of diabetes, and $\mathrm{HbA}_{1 \mathrm{c}}$ values before the test as the independent variables. We found a significant negative correlation $(r=0.59)$ between amplitudes and age at onset $(p=0.002)$ and duration of disease $(p=0.002)$ at only 1.4 cycles/deg spatial frequency. The $\mathrm{HbA}_{\mathrm{Ic}}$ values were not correlated. 


\section{Spatial frequency (cycles / deg)}

0.6
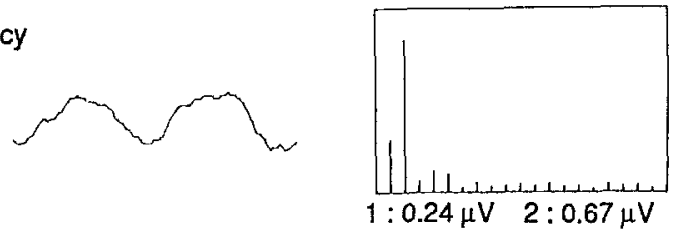

1.0
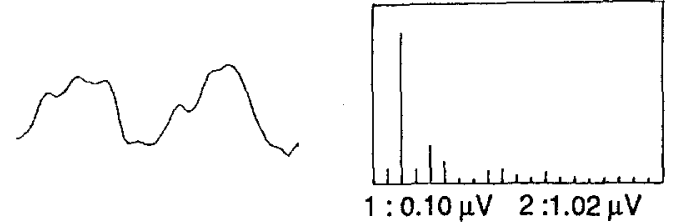

1.4
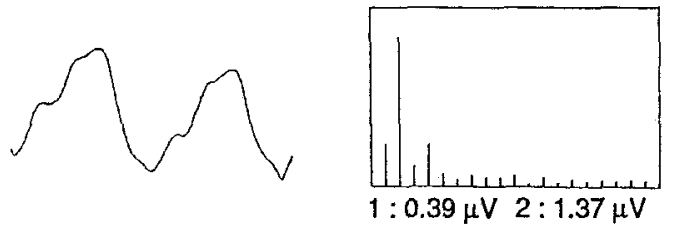

2.2

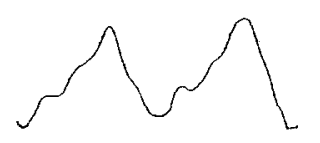

4.8

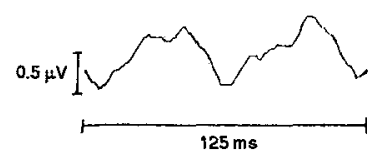

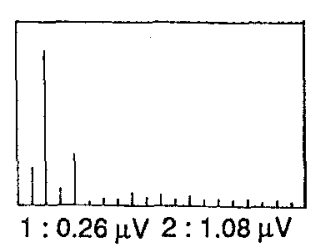

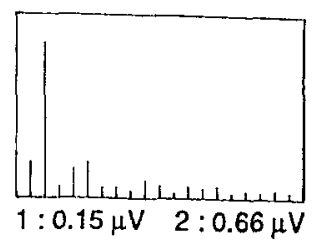

Fig. 2. Representative example of waveforms (left column) and corresponding Discrete Fourier Series (right column) of a steady state pattern electroretinogram (P-ERG) as a function of stimulus spatial frequency in a control subject. Absolute amplitude numerical values $(\mu V)$ of 1 st and 2 nd P-ERG components (left and right respectively) are also reported for each of the tested spatial frequencies. Note that P-ERG is essentially dominated by the 2 nd harmonic component ( $2 \mathrm{nd}$ bar from left) in each case. The highest amplitude value of the 2 nd component $(1.37 \mu \mathrm{V})$ can be observed at 1.4 cycles/deg stimuli
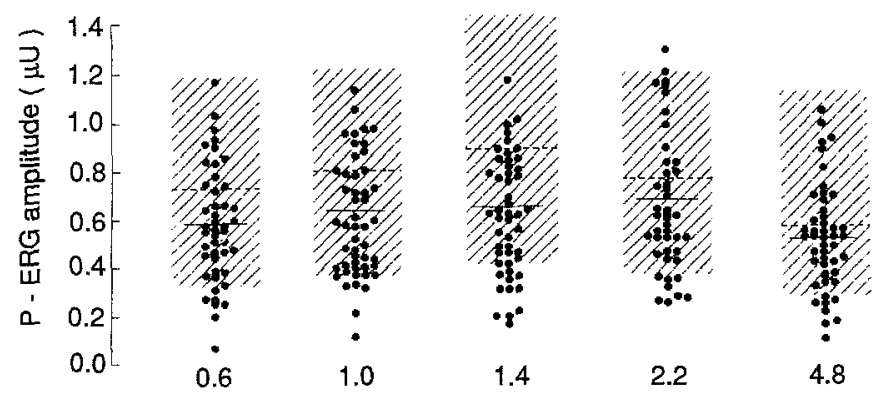

Spatial frequency (cycles / deg )

Fig.3. Pattern electroretinogram amplitude as a function of spatial frequency in each eye of diabetic patients. Shaded areas indicate normal ranges of control subjects at corresponding spatial frequencies. The normality test of control groups was not significant. Horizontal bars represent the means of diabetic patients. Normal means (dashed bars) are also indicated

\section{Discussion}

Visual function has recently been receiving increased attention for its use in detecting neurosensory deficits in diabetic patients with no or early retinopathy [19]. An abnormal electroretinal function can be detected by oscillatory potentials, conventional uniform-field flashERG [4, 5], and P-ERG [8, 9, 20, 21]. However, flashERG responses reflecting the activity of the entire retina do not evaluate macular function as well as the P-ERG. The P-ERG can be useful to directly estimate the activity of the central retinal area [12]. P-ERG abnormalities can be observed in ganglion cell dysfunctions [22-24]. However, the integrity of more outer retinal elements is also required for P-ERG generators. An impairment of photoreceptors, bipolar cells, amacrine cells, and horizontal cells may contribute to P-ERG reductions. Although a dysfunction of all inner retinal cells cannot be excluded, the interference with normal excitation from cone cells to their ganglion cells seems to be unlikely. In fact, receptor function in early diabetes [25] and in disorders affecting selectively ganglion cells [26] has been found to be normal.

Previous studies $[8,9,20,21]$ have not systematically evaluated the amount of P-ERG reductions as a function of spatial frequency. Moreover, abnormal P-ERG responses were observed in diabetic patients after the appearance of the microvascular retinal damage. In this study, we recorded the steady state P-ERG as a function of this stimulus parameter to evaluate possible selective dysfunctions in early diabetes. P-ERG spatial selectivity depends on the specific stimulation and/or recording technique used to obtain the response and can only be demonstrated under a relatively narrow range of stimulus conditions [27, 28]. The use of fast-reversing gratings rather than slow-reversing checkerboards as a stimulus $[14,15]$ combined with Fourier analysis of the steadystate responses [17] may help reveal P-ERG spatial selectivity as well as improve the diagnostic sensitivity of this technique.

Retinal ganglion cells functionally exhibit different characteristics of their receptive fields depending on soma and dendritic tree size $[29,30]$. Cells with large receptive fields are better stimulated by lower spatial frequencies, whereas ganglion neurons with small receptive fields are preferentially stimulated by higher spatial frequencies. PERG generators can show a selective vulnerability to several diseases (e.g., early glaucoma [31] and optic neuritis [14]), in which spatial frequency-dependent P-ERG abnormalities have been found.

In early diabetes, our results show that a P-ERG selective impairment might be attributed to a dysfunction of subpopulations of retinal ganglion cells. Spatial frequency-dependent losses [1-3] have been shown by using contrast sensitivity gratings and Snellen letter charts (i. e., a test employing white cardboard with black letters) in diabetic patients only after the onset of clinically detectable retinopathy. Regan and Neima [3] hypothesized that ganglion cells with a large receptive field were more vulnerable to functional loss, since a great number of the dendritic processes could more easily lose their ability to 
transmit neural signals [32]. Our results show that the use of alternating gratings with our electroretinographic procedure, rather than that with subjective psychophysical tests yields a greater number of functional losses which may occur in the inner retinal layers.

These retinal dysfunctions, whose mechanisms are not fully understood, are probably due to insulin deficiency and/or hyperglycaemia that play a fundamental role in the development of diabetic retinopathy. Very slowly progressive metabolic alterations develop after a long latent period until clinically detectable diabetic retinopathy finally appears. A recent interesting study in diabetic rabbits demonstrated that the biochemical mechanisms underlying the functional disturbances cannot be secondarily attributed to changes in the microcirculation because the rabbit retina is avascular [33]. Although the predictive value of impaired ganglion cell function for either the subsequent development or clinical course of diabetic retinopathy has not been established, P-ERG reductions indicate an increasing vulnerability to the development of retinopathy.

In this study, P-ERG responses were inversely correlated with age at onset of diabetes. This result is consistent with previous findings in which P-ERG amplitude is lower in older than younger normal subjects [34].P-ERG amplitudes were also correlated with duration of diabetes only at the peak spatial frequency $(1.4$ cycles/deg). Early lesions can be associated with the duration of the disease [35]. The course of diabetes may progressively increase the vulnerability to the development of vascular injuries, according to P-ERG amplitude reductions.

In conclusion, our findings indicate spatial frequencydependent abnormalities of the P-ERG in Type 1 diabetic patients who have no signs of retinopathy. This suggests a selective vulnerability of inner retina elements (i.e., ganglion cells with larger receptive fields) which respond preferentially to medium and large size stimuli.

Acknowledgements. We are grateful to L.Buzzonetti, F. M.Cefalà, and $\mathrm{G}$. Porrello for their technical assistance.

\section{References}

1. Ghafour MI, Fould WS, Allan D, McClure E (1982) Contrast sensitivity in diabetic subjects with and without retinopathy. Br J Ophthalmol 66: 492-495

2. Sokol S, Moskowitz A, Skarf B, Evans R, Molitch M, Senior B (1985) Contrast sensitivity in diabetics with and without background retinopathy. Arch Ophthalmol 103: 51-54

3. Regan D, Neima D (1984) Low-contrast letter charts in early diabetic retinopathy, ocular hypertension, glaucoma, and Parkinson's disease. Br J Ophthalmol 68: 885-889

4. Simonsen SE (1980) The value of oscillatory potential in selecting juvenile diabetics at risk of developing proliverative retinopathy. Acta Ophthalmol 58: 865-878

5. Bresnick GH, Palta M (1987) Predicting progression to severe proliferative diabetic retinopathy. Arch Ophthalmol 105: $810-814$

6. Pozzessere G, Rizzo PA, Valle E, Mollica MA, Meccia A, Morano S, Di Mario U, Andreani D, Morocutti C (1988) Early detection of neurological involvement in IDDM and NIDDM: multimodal evoked potentials versus metabolic control. Diabetes Care 11: 473-480
7. Algan M, Ziegler O, Gehin P, Got I, Raspiller A, Weber M, Genton P, Saudax E, Drouin P (1989) Visual evoked potentials in diabetic patients. Diabetes Care 12:227-229

8. Arden GB, Hamilton AMP, Wilson-Holt J, Ryan S, Yudkin JS, Kurtz A (1986) Pattern electroretinogram become abnormal when background diabetic retinopathy deteriorates to a preproliferative stage: possible use as a screening test. $\mathrm{Br} \mathrm{J}$ Ophthalmol 70: $330-335$

9. Coupland SG (1987) A comparison of oscillatory potential and pattern electroretinogram measures in diabetic retinopathy. Doc Ophthalmol 66: 207-217

10. Fiorentini A, Maffei L, Pirchio M, Spinelli D, Porciatti V (1981) The ERG in response to alternating gratings in patients with diseases of the peripheral visual pathways. Invest Ophthalmol Vis Sci 21: 490-493

11. Hess RF, Baker CJ (1984) Human pattern-evoked electroretinogram. J Neurophysiol 51:939-951

12. Maffei L, Fiorentini A (1986) Generator sources of the pattern ERG in man and animals. In: Cracco RQ, Bodis-Wollner I (eds) Frontiers in clinical neuroscience, vol3. Liss, New York, pp 101-116

13. Maffei L, Fiorentini A (1981) Electroretinographic responses alternating gratings before and after section of the optic nerve. Science 211: 953-955

14. Porciatti V, Berger GP von (1984) Pattern electroretinogram and visual evoked potential in optic nerve disease: early diagnosis and prognosis. Doc Ophthalmol Proc Ser 40: 117-126

15. Porciatti V (1987) Non-linearities in the focal ERG evoked by pattern and uniform-field stimulation. Their variation in retinal and optic nerve dysfunction. Invest Ophthalmol Vis Sci 28: $1306-1313$

16. Korth M, Rix R (1985) Changes in spatial selectivity of patternERG components with stimulus contrast. Graefes Arch Clin Exp Ophthalmol 223: 23-28

17. Fadda A, Falsini B, Neroni M, Porciatti V (1989) Development of a personal computer software for a visual electrophysiology laboratory. Comput Methods Programs Biomed 28: 45-50

18. Ray WA, O'Day DM (1985) Statistical analysis of multi-eye data in ophthalmic research. Invest Ophthalmol Vis Sci 26: 1186-1188

19. Bresnick GH (1986) Diabetic retinopathy viewed as a neurosensory disorder. Arch Ophthalmol 104: 989-990

20. Wanger P, Persson HS (1985) Early diagnosis of retinal changes in diabetes: a comparison between electroretinography and retinal biomicroscopy. Acta Ophthalmol 63: 716-720

21. Boschi MC, Frosini R, Mencucci R, Sodi A (1989) The influence of early diabetes on the pattern electroretinogram. Doc Ophthalmol 71: 369-374

22. May JG, Ralston JV, Reed JL, Dike HJ van (1982) Loss in pattern-elicited electroretinograms in optic nerve dysfunction. Am J Ophthalmol 93: 418-422

23. Wanger P, Persson HE (1983) Pattern-reversal electroretinograms in unilateral glaucoma. Invest Ophthalmol Vis Sci 24 $749-753$

24. Bodis-Wollner I, Bobak P, Harnois C, Mylin L (1984) Methodological aspects of signal-to-noise evaluation of simultaneous pattern electroretinogram and visual evoked potential recording. Doc Ophthalmol Proc Ser 40: 39-48

25. Caputo S, Di Leo MAS, Ghirlanda G, Falsini B, Marietti G, Porciatti V, Greco AV (1989) Steady-state focal electroretinogram in Type 1 (insulin-dependent) diabetes with no or early retinopathy. Diabetologia 32: 472-473 (Abstract)

26. Porciatti V, Falsini B, Fadda A, Bolzani R (1989) Steady-state analysis of the focal ERG to pattern and flicker: relationship between ERG components and retinal pathology. Clin Vision Sci 4: 323-332

27. Odom VJ, Maida TM, Dawson WW (1982) Pattern evoked retinal responses (PEER) in human: effects of spatial frequency, temporal frequency, luminance and defocus. Curr Eye Res 2: 99-108

28. Sokol S, Jones K, Nadler D (1983) Comparison of the spatial response properties of the human retina and cortex by simulta- 
neously recorded pattern ERGs and VEPs. Vision Res 7: 723-727

29. Boycott BB, Wässle H (1974) The morphological types of ganglion cells of the domestic cat's retina. J Physiol (London) 240: 397-419

30. Monasterio de FM (1978) Properties of concentrically organized $\mathrm{X}$ and $\mathrm{Y}$ ganglion cells of the retina of macaques. J Neurophysiol 41: 1394-1417

31. Porciatti V, Falsini B, Brunori S, Colotto A, Moretti C (1987) Pattern electroretinogram as a function of spatial frequency in ocular hypertension and early glaucoma. Doc Ophthalmol 65: 349-355

32. Regan D, Neima D (1984) Balance between pattern and flicker sensitivities in the visual fields of ophthalmological patients. Br J Ophthalmol 68: 310-315

33. MacGregor IC, Rosecan LR, Laties AM, Matschinsky FM (1986) Altered retinal metabolism in diabetes. I. Microanalysis of lipid, glucose, sorbitol, and myo-inositol in the choroid and in the individual layers of the rabbit retina. J Biol Chem 261: $4046-4051$
34. Porciatti V, Falsini B, Scalia G, Fadda A, Fontanesi G (1988) The pattern electroretinogram by skin electrodes: effect of spatial frequency and age. Doc Ophthalmol 70: 117-122

35. Pirart J (1978) Diabetes mellitus and its degenerative complications: a prospective study of 4,400 patients observed between 1947 and 1973. Diabetes Care 1: 168-188

Received: 27 February 1990

and in revised form: 15 June 1990

Dr. M.A.S.Di Leo

Università Cattolica del Sacro Cuore

Istituto di Clinica Medica

Largo A. Gemelli, 8

I-00168 Roma

Italy

\section{Announcements}

\section{Keystone Symposia, 1991 Conferences}

The Adipose Cell: A Modell for Integration of Hormone Signalling in the Regulation of Cellular Function

This symposium will take place from January 18-24, 1991 in Park City, Utah.

\section{Diabetes and Insulin Action}

This symposium will take place from January 18-24, 1991 in Park City, Utah.

\section{Gene Regulation and Signalling in Endocrine Systems}

This symposium will take place from January 19-25, 1991 at Steamboat Springs, Colorado.
For more information on the above symposia please contact: Keystone Symposia, 2032 Armacost Avenue, Los Angeles, CA 90025, USA. Tel: (213) 207-5042, Fax: (213) 207-2397, Telex: 495012 attn: Keystone Symposia

\section{The British Council}

Diabetes mellitus: control and complications - an interactive workshop to be held at Guy's Hospital, London, from 17-22 February 1991. For more information please contact: the nearest British Council Office or Courses Department, the British Council, 65 Davies Street, London W1Y 2AA, UK. Applicants are advised to apply before 18 November 1990. Tel: 071-9308466

\section{Erratum}

\section{Diabetologia, Volume 33, Number 9, September 1990, pp. 557-560}

S. Assa and C.Moroz: Elevated serum placental isoferritin in newly diagnosed Typ 1 (insulin -dependent) diabetes mellitus. A possible marker for identification of high risk subjects.
An error occurred in the introduction of the above paper. In the first paragraph, third sentence, the percentage range was wrong. Instead of " $30-50 \%$ ", it should have been: "Some of these autoantibodies are observed in $50-90 \%$ of newly diagnosed Type 1 diabetes patients and in $3-5 \%$ of their first degree relatives $[1-5]$. 